\title{
Definição de Envelhecimento Saudável na Perspectiva de Indivíduos Idosos
}

\author{
Successful Aging Definition in a Sample of Older Adults
}

\author{
Ana Paula Fabrino Bretas Cupertino*a, Fernanda Heringer Moreira Rosa*b \\ \& Pricila Cristina Correa Ribeiro*c \\ ${ }^{\mathrm{a}}$ Kansas University Medical Center, Kansas City, USA, ${ }^{\mathrm{b}}$ Universidade Estadual de Campinas, Campinas, Brasil, \\ ${ }^{\mathrm{c}}$ Universidade Estadual de Campinas, Campinas, Brasil
}

\begin{abstract}
Resumo
O envelhecimento é definido como um processo multidimensional (Baltes \& Baltes, 1990). Rowe e Kahn (1998) apresentam três indicadores de envelhecimento saudável: baixo risco de doenças e de incapacidades funcionais; funcionamento mental e físico excelentes; e envolvimento ativo com a vida. Com base nesses pressupostos, o objetivo deste trabalho foi examinar a definição de envelhecimento saudável em uma amostra de idosos da comunidade buscando identificar as multidimensões percebidas pelos mesmos. A amostra foi de 501 idosos com idade entre 60 a 93 anos $(M=72,65 ; D P=8,08)$. Foi realizada análise de conteúdo das respostas abertas sobre envelhecimento saudável. Identificou-se 29 categorias, sendo as mais citadas: saúde física; saúde social e saúde emocional e as menos citadas: envelhecimento como patológico e atividades específicas para idosos. Conclui-se que este estudo confirmou os pressupostos de heterogeneidade e multidimensionalidade do processo de envelhecimento, ao observar-se a variabilidade nas definições dos idosos.

Palavras-chave: Envelhecimento saudável; análise qualitativa; multidimensionalidade.
\end{abstract}

\begin{abstract}
The process of aging is defined as a multidimensional process (Baltes e Baltes, 1990). Rowe and Kahn (1998) present three indicators of successful aging: low risk of disease and disease-related disabilities; high level of mental and physical functions and an active life style. Based on these presumptions, this article presents a research that examined the definition of successful aging from a sample of older adults, seeking to identify the multiple dimensions perceived by them. The sample consisted of 501 older adults between 60 to 93 years old $(\mathrm{M}=72,65 ; \mathrm{SD}=8,08)$. Content analysis of their answers to what successful aging means identified 29 possible categories. The most cited definitions for successful aging were: physical health, social health and emotional health. On the other hand, the least cited answers were: a life of work; aging as a pathological process and specific activities for older adults. In conclusion, this study confirmed the presumptions of heterogeneity and multidimensionality of aging, by observing the various definitions from older adults.

Keywords: Successful aging; qualitative analysis; multidimensionality.
\end{abstract}

A reflexão sobre as modificações no perfil etário tem gerado reformulações e ampliações teórica e empíricas no campo da psicologia desenvolvimentista e do envelhecimento. Até início do século XX, predominavam mitos e estereótipos sobre o idoso influenciando a sociedade e comunidade acadêmica. Priorizava-se estudos nas faixas etárias da infância e adolescência, e outros poucos comparando performance entre grupos de adultos e idosos, o que resultava na vinculação do envelhecimento à perdas e involução no desenvolvimento.

Entretanto, em 1903, Elie Metchnikoff enfatizou a importância da Gerontologia no campo da saúde. Em 1922, o psicólogo Stanley Hall defendeu que existiriam diferenças no sentir e pensar dos idosos e que também haveria varia-

\footnotetext{
* Endereço para correspondência ( $1^{\circ}$ autor): Department Preventive Medicine University of Kansas Med Center. 3901. Rainbow Blvd. 4028 Varnes Kansas City-KS-66160. Fones: (913) 588-2783 - Fax: (913) 588-8398. E-mail: heringer_psi@yahoo.com.bre priccr@yahoo.com.br

Agradecimentos ao financiamento do CNPq projeto PROFIX processo 54.0956/ $001-5 \mathrm{NV}$.
}

ções entre os indivíduos da mesma faixa etária. Outra importante contribuição foi dada por Charlotte Bühler que em 1935, com os resultados das análises de 300 autobiografias de homens adultos, concluiu que o desenvolvimento psicológico seria um processo baseado em metas e caracterizado pela extensão, nos anos da infância e da adolescência e a contração nos anos da maturidade e da velhice (Neri, 1997). Apenas em meados do século XX, fruto do investimento em estudos longitudinais e de coorte seqüencial, foi possível examinar a heterogeneidade no processo do envelhecimento, marcando assim o fim da busca pela normatização e o início da investigação dos determinantes do envelhecimento saudável. Dessa forma, a atual literatura gerontológica discute alguns conceitos, de forma a enfatizar o que seria importante para se obter um envelhecimento saudável.

A perspectiva do Curso da Vida é central na proposta da Psicologia do Envelhecimento. Tal abordagem começou a firmar-se a partir dos anos 70 e enfatiza conceitos como plasticidade humana, multidirecionalidade, multidimensionalidade, resiliência e múltiplas trajetórias 
de vida. Staudinger, Marsiske e Baltes (1995) defendem que as perdas no envelhecimento podem ser compensadas por meio dos níveis de reservas e da capacidade de resiliência, e os ganhos podem ser obtidos com a seleção e otimização das competências geradas pelo envelhecimento.

Explorando a variabilidade no desenvolvimento humano, Rowe e Kahn (1998) propõem três trajetórias do envelhecimento humano: normal, patológica e saudável. A definição de envelhecimento saudável proposta por estes autores prioriza baixo risco de doenças e de incapacidades funcionais relacionadas às doenças; funcionamento mental $\mathrm{e}$ físico excelentes; e envolvimento ativo com a vida.

Uma outra teoria que traz sua contribuição para os estudos referentes ao envelhecimento, é a teoria da transcendência do ego, cuja concepção está vinculada a idéia de que o envelhecimento está inserido no aspecto dialético da finitude e da permanência, onde o indivíduo seria conduzido a sua essência tornando-se livre do determinismo culturais e ambientais (Levenson, Aldwin \& Cupertino, 2001).

Aldwin (1994) argumenta que os idosos, frente aos eventos de perda, lidam muito bem com o estresse, ou seja, os idosos geralmente são mais eficientes do que os adultos mais jovens em suas respostas de enfrentamento ao estresse. A autora explica estes dados em função de eventos considerados marcantes vividos ao longo de toda a vida ampliando a possibilidade de que, no envelhecimento, os idosos possuam tanto um maior repertório de estratégias de enfrentamento quanto o fato de que estes tornam-se mais eficazes com o passar dos anos.

A sabedoria é outro importante aspecto abordado quando o tema é envelhecimento saudável, Baltes e Smith (1995) a definem como uma função pertinente ao crescimento ao longo da vida, uma especialização cognitiva rara e difícil de ser atingida. Assim, a sabedoria seria o resultado do processo de revisão de vida e seria alcançada, principalmente, na velhice, sendo considerada um dos maiores ganhos dessa fase da vida, uma vez que permite ao indivíduo ir além das perdas vividas no processo de envelhecimento (Mauad, 1999).

As evoluções nos paradigmas sobre o desenvolvimento e o envelhecimento, trazem para discussão a possibilidade do envelhecimento poder ser vivido com satisfação, saúde e bem-estar, instigando a busca de variáveis que interferem no alcance de um envelhecimento bem-sucedido. Atualmente, os pesquisadores buscam definir o envelhecimento saudável, ou bem-sucedido, entretanto, observa-se que poucos investigaram como os próprios idosos definem o envelhecimento ideal e como consideram a possibilidade de se alcançar esse envelhecimento. Sendo assim, o objetivo do presente trabalho é examinar em amostra de idosos na comunidade a definição de envelhecimento saudável entre os mesmos, e mais especificamente, relacionando o conteúdo das respostas entre gênero e idade.

\section{Amostra}

\section{Método}

Este estudo utilizou uma sub-amostra de 501 idosos entrevistados no Projeto Processos do Envelhecimento Saudável (PENSA), realizado com 956 residentes da cidade de Juiz de Fora/ MG. A sub-amostra corresponde a indivíduos que responderam às perguntas qualitativas sobre a definição de envelhecimento saudável e meios de alcançar esse envelhecimento. A idade variou entre 60 a 93 anos de idade $(M=72,65 ; D P=8,08)$. Sendo $39 \%$ idoso-jovem (60-69 anos); 41\% idoso-médio (70-89 anos) e 20\% idodoidoso (acima de 90 anos). Quanto ao gênero, $71 \%$ da amostra é constituída por mulheres.

\section{Instrumentos}

Foi utilizada a entrevista semi-estruturada realizada pelo projeto PENSA, da qual foram utilizadas as variáveis para o presente estudo. Analizou-se variáveis sócio-demográficas (sexo e idade) e duas perguntas abertas sobre a definição de envelhecimento saudável: (a) O que é o envelhecimento saudável na sua opinião? e (b) O que você acha que é importante para ter um envelhecimento saudável?

\section{Procedimentos}

Observou-se, a partir da revisão teórica, que o envelhecimento saudável é um processo multidimensional, o qual envolve aspectos físicos, sociais, cognitivos e emocionais (Aldwin, 1994; Baltes \& Baltes, 1990; Baltes \& Smith, 1995; Levenson, Aldwin \& Cupertino, 2001; Mauad, 1999; Neri, 1997; Rowe \& Kahn, 1998). Com base nesta revisão foram elaboradas 20 categorias englobando múltiplas dimensões do envelhecimento saudável (saúde física; saúde social; saúde emocional; saúde cognitiva; alimentação e exercícios; evitar fatores de risco; atividades gerais; aceitação dessa fase; espiritualidade; atividade operacional; estrutura familiar; viver plenamente; ter espírito jovem; ter novas habilidades; não guardar mágoas; aprender; integridade; manter o controle; atividades específicas para a terceira idade; envelhecimento patológico). Posteriormente, foram incluídas outras nove categorias baseadas nas respostas dos idosos (não existe envelhecimento saudável; não saber definir; pouca ambição na vida; influência dos pais; estabilidade financeira; beleza física; não ter problema; fazer o bem; ter trabalhado ao longo da vida). Dessa forma, partindo do estudo teórico e do relato das respostas manifestas pelos idosos, foi elaborado um roteiro com 29 categorias concernentes à definição de envelhecimento saudável (Tabela 1) na próxima página.

Baseando-se nas 29 categorias, dois juízes realizaram a análise qualitativa utilizando o método de definição das categorias, que consiste no agrupamento de conteúdos de forma que seus elementos possuam um significado constitutivo (Bardin, 1977/1979). Foram seguidos os critérios: (a) homogeneidade entre as escolhas das respostas nas categorias; (b) esgotamento do texto; (c) exclusividade do conteúdo em somente uma categoria; (d) objetividade na avaliação; (e) regras adaptadas ao conteúdo e ao objetivo do trabalho (exemplo de comunicação dos idosos, ver Tabela 2 na págian 87). Para verificação da confiabilidade entre os juízes foi utilizado o programa SPSS o qual demonstrou altos índices de confiabilidade entre os juizes, Alpha Cronbach variando entre 0.80 e 1.00 . 
Quanto a análise estatística dos dados, realizou-se análise univariada para quantificação da freqüência das categorias apontadas pelos idosos. Para avaliar a relação entre as categorias e as variáveis gênero e idade, utilizou-se o teste do Qui-quadrado. Essas análises foram realizadas utilizando-se o software estatístico SPSS 10.0.

\section{Resultados}

Nos resultados da análise da definição de envelhecimento saudável e o que é importante para se obter esse envelhecimento, as categorias mais apontados pelos idosos, como mostra a Tabela 1, foram: saúde física (53\%), saúde social (46\%), saúde emocional (37\%), preocupação com alimentação/ exercícios (36\%) e evitar fatores de risco (19\%).

Quanto aos aspectos menos enfatizados pelos idosos na definição de envelhecimento saudável têm-se: atividadesespecificas para $3^{a}$ idade (0.4\%), trabalhar ao longo da vida $(0.6 \%)$, envelhecimento visto como patológico (0.6\%) e aprender com os pais $(0.8 \%)$ (Tabela 1).

Tabela 1

Percentual das Categorias Citadas pelos Idosos

\begin{tabular}{|c|c|c|c|}
\hline Categorias & $\%$ & Categorias & $\%$ \\
\hline - saúde física & 53 & - ter espírito jovem & 5 \\
\hline • saúde social & 46 & - ter novas habilidades & 5 \\
\hline • saúde emocional & 37 & • não guardar mágoas & 4 \\
\hline - alimentação, exercícios & 36 & - aprender coisas novas & 3 \\
\hline - evitar fatores de risco & 19 & - integridade & 3 \\
\hline - atividades gerais & 18 & - não existe envelhecimento & 2 \\
\hline • aceitação dessa fase & 18 & • não sabe definir & 2 \\
\hline - ter fé, espiritualidade & 15 & • pouca ambição & 2 \\
\hline - atividade operacional & 13 & - influência dos pais & 1 \\
\hline • saúde cognitiva & 12 & - manter o controle & 1 \\
\hline • estrutura familiar & 12 & • beleza física & 1 \\
\hline - estabilidade financeira & 9 & - envelhecimento patológico & 0.6 \\
\hline • não ter problemas & 9 & - ter trabalhado & 0.6 \\
\hline - fazer o bem & 8 & - atividade específica & 0.4 \\
\hline - viver plenamente & 6 & • outros & 17 \\
\hline
\end{tabular}

Os resultados da correlação entre categorias e gênero (Tabela 3 ) na página 88 , apontam uma relação significativa do gênero com a categoria estabilidade financeira, $X^{2}(1)=$ $21.8 ; p<.000$, dos $9 \%$ dos idosos que apontaram esta categoria $5 \%$ eram homens. Ressalta-se a categoria não guardar mágoas apontada somente por mulheres, com significância de $X^{2}(1)=6.23 ; p<.01$.

Ao considerar a relação entre categorias e idade, como ilustra a Tabela 3, foram obtidas correlações significativas com as categorias saúde emocional, dos $37 \%$ que citaram esta categoria, $16 \%$ estão no grupo idoso-jovem e $16 \%$ no idoso-médio, $X^{2}(2)=7.72 ; p<0.05$; alimentação e exercícios fïsi$\cos$ foi citada por $36 \%$ da amostra, sendo $16 \%$ do grupo idoso-jovem, $X^{2}(2)=6.26 ; p<0.05$. Dos $9 \%$ que apontaram a categoria estabilidade financeira, $5 \%$ pertencem ao grupo idoso-jovem, $X^{2}(2)=8.03 ; p<0.05$, nota-se também que $2 \%$ da amostra não soube definir o envelhecimento, deste total, $1 \%$ pertence ao grupo idoso-idoso, $X^{2}(2)=7.65 ; p<0.05$.
A presente análise indica ainda uma tendência de que as mulheres apontem mais do que os homens as categorias saúde social, $X^{2}(1)=2.497 ; p<0.1 ;$ saúde emocional, $X^{2}(1)=2.74 ; p<0.1$, espiritualidade, $X^{2}(1)=3.43 ; p<0.1 ;$ atividade operacional, $X^{2}(1)=2.31 ; p<0.1 ;$ manter espirito jovem, $X^{2}(1)=3.31 ; p<0.1 ;$ integridade $X^{2}(1)=2.53 ; p<0.1$ e $n \tilde{a} o$ soube definir $X^{2}(1)=2.90 ; p<0.1$. Considerando ainda a análise entre as categorias e idade, nota-se uma tendência de que o idoso-jovem e idoso-médio indiquem em maior proporção a categoria atividades gerais, $X^{2}(1)=4.11 ; p<0.1 \mathrm{e}$ integridade tendendo a um maior enfoque entre o idosojovem $X^{2}(2)=5.50 ; p<0.1$.

\section{Discussão}

Os resultados da análise de conteúdo da opinião dos idosos sobre envelhecimento saudável demonstram baixa incidência de opiniões considerando o envelhecimento como patológico (0.6\%), corroborando com as definições de envelhecimento saudável defendidas pela Psicologia do Envelhecimento e demonstrando modificações nos paradigmas de velhice enquanto fase de perdas e declínios.

Quanto aos aspectos mais apontados pela amostra em sua definição de envelhecimento saudável, destaca-se a saúde fisica, demonstrando que para os idosos a manutenção da saúde física é fundamental para um envelhecimento saudável. Sabendo-se que o processo de envelhecimento físico acarreta modificações anatômico-fisiológicas que implicam na necessidade de adaptações e compensações para um menor desequilíbrio entre ganhos e perdas (Staudinger, Marsisker \& Baltes, 1995), pode-se identificar, na amostra, uma transcendência do paradigma de envelhecimento ideal enquanto ausência de perdas físicas, visto que a mesma tende a enfocar a aceitação da velhice como sinônimo de envelhecimento saudável.

Quanto aos resultados da correlação entre as categorias, observa-se falta de correlação na categoria manter-se com espírito jovem, o que pode indicar a crença de que o envelhecimento saudável está relacionado a compensar perdas físicas, sociais e cognitivas em prol da manutenção da jovialidade do espírito. A falta de correlação da categoria espiritualidade pode ser explicado pelo fato dos idosos, que enfatizaram essa categoria, considerarem a interferência divina como principal responsável pela trajetória do envelhecimento, não se preocupando em destacar aspectos como controle, fatores externos e mesmo manutenção da saúde.

É importante destacar a correlação entre saúde emocional e integridade, demonstrando que a comunicação dos idosos confirma a literatura sobre a importância da revisão de vida e alcance da integridade na determinação de um processo saudável de envelhecimento (Erikson, Erikson \& Kivnick, 1986, apud Papalia \& Olds, 2000).

Também foi encontrada correlação entre as categorias evitar fatores de risco e a a doção de comportamentos preventivos como alimentação e exercícios, demonstrando que os idosos que destacam o risco também enfatizam a prevenção. 
Tabela 2

Exemplos da Comunicação dos Idosos por Categoria

\begin{tabular}{|c|c|}
\hline Categorias & Comunicação dos idosos \\
\hline $\begin{array}{l}\text { - saúde cognitiva, saúde física; } \\
\text { atividades gerais }\end{array}$ & $\begin{array}{l}\text { - "Além de ter saúde, continuar } \\
\text { tendo uma vida ativa, uma } \\
\text { boa cabeça." }\end{array}$ \\
\hline $\begin{array}{l}\text { - suporte social; atividade } \\
\text { operacional }\end{array}$ & $\begin{array}{l}\text { • “... acho que o trabalho faz } \\
\text { muita falta para o idoso... ser } \\
\text { mais ativo." }\end{array}$ \\
\hline $\begin{array}{l}\text { • saúde emocional; sem mágoas; } \\
\text { fé; integridade }\end{array}$ & $\begin{array}{l}\text { • "Viver sem se queixar, ter fé } \\
\text { em Deus e combater os pro- } \\
\text { blemas... não ter raiva." }\end{array}$ \\
\hline $\begin{array}{l}\text { - alimentação, exercícios; evi- } \\
\text { tar fatores de risco }\end{array}$ & $\begin{array}{l}\text { • “... não beber, não fumar, não } \\
\text { ficar sem dormir e não comer } \\
\text { mal.” }\end{array}$ \\
\hline $\begin{array}{l}\text { - estabilidade financeira; estru- } \\
\text { tura familiar }\end{array}$ & $\begin{array}{l}\text { - "Não ter problema financeiro, } \\
\text { muito menos com a família. } \\
\text { É viver bem com a família." }\end{array}$ \\
\hline $\begin{array}{l}\text { • não ter problemas; aprender } \\
\text { com os pais }\end{array}$ & $\begin{array}{l}\text { • "Não se aborrecer muito... } \\
\text { obedecer aos pais, receber } \\
\text { conselhos." }\end{array}$ \\
\hline $\begin{array}{l}\text { - pouca ambição; viver plena- } \\
\text { mente cada dia }\end{array}$ & $\begin{array}{l}\text { • “... não ficar lastimando pen- } \\
\text { sando no passado. Olhar pra } \\
\text { frente... viver com entusiasmo, } \\
\text { como se fosse o primeiro dia." }\end{array}$ \\
\hline $\begin{array}{l}\text { - aprender coisa nova; trabalhar } \\
\text { ao longo da vida }\end{array}$ & $\begin{array}{l}\text { •"Ser trabalhador responsável, } \\
\text { respeitar os outros e não ter } \\
\text { preocupação." }\end{array}$ \\
\hline $\begin{array}{l}\text { - atividades para } 3^{\text {a }} \text { idade; } \\
\text { manter o controle }\end{array}$ & $\begin{array}{l}\text { - "Aproveitar a vida, dançar, ir } \\
\text { a AMAC." }\end{array}$ \\
\hline • jovialidade; beleza física & $\begin{array}{l}\text { •“... conversar com os jovens } \\
\text { para não sentir a idade bioló- } \\
\text { gica que tenho.” }\end{array}$ \\
\hline - fazer o bem & $\begin{array}{l}\text { • "Fazer o bem e respeitar o } \\
\text { próximo." }\end{array}$ \\
\hline - sabedoria; integridade & $\begin{array}{l}\text { - "Olho pra traz e nada me faz } \\
\text { sofrer, porque fiz o melhor } \\
\text { que podia..." }\end{array}$ \\
\hline $\begin{array}{l}\text { • aceitação “dessa fase”; desen- } \\
\text { volver habilidade }\end{array}$ & $\begin{array}{l}\text { • "É conseguir aceitar melhor } \\
\text { os limites que o envelheci- } \\
\text { mento impõe." }\end{array}$ \\
\hline $\begin{array}{l}\text { - envelhecimento patológico; } \\
\text { não existe saúde; não sabe } \\
\text { definir envelhecimento sau- } \\
\text { dável }\end{array}$ & $\begin{array}{l}\text { • "Acho que não existe... enve- } \\
\text { lhecimento é só doença e tris- } \\
\text { teza." }\end{array}$ \\
\hline
\end{tabular}

Entretanto, ao contrário do que se poderia pensar, estas categorias não se correlacionaram com a ênfase na saúde física, demonstrando que aqueles que destacam a saúde física não se preocupam com as questões de prevenção e risco. Por outro lado, a saúde física correlacionou-se com a estabilidade financeira e saúde cognitiva, expressando que, segundo essa amostra, a manutenção da saúde está associada à manutenção das capacidades cognitivas. Possivelmente a manutenção da saúde foi vinculada a questão financeira, por depender desta em aspectos instrumentais tais como a compra de remédios, a ida ao médico, dentre outros.

A saúde física também correlacionou-se com a categoria atividades gerais, indicando que um dos critérios utilizados pelos idosos para a avaliação de sua saúde física permeia-se em sua auto-avaliação da manutenção das atividades de vida diária, pressuposto este também abordado na atual concepção de qualidade de vida, pois envolve aspectos multidimensionais que englobam as percepções das pessoas sobre o significado de suas próprias vidas (Paschoal, 2002; Ramos, 2002).

Destaca-se ainda a correlação entre saúde social e estrutura familiar, expressando que a família representa tanto um suporte social para os idosos quanto uma preocupação de bem-estar dos mesmos, com os membros da família, ou seja, a amostra tende a definir envelhecimento saudável na possibilidade de vislumbrar um bem-estar amplo dos filhos e parentes, observando aspectos emocionais, físicos e financeiros, sendo este último comprovado pela correlação positiva entre as categorias estrutura familiar e estabilidade financeira.

$\mathrm{Na}$ diferença encontrada nas relações entre gênero e categorias, observou-se predominância de homens apontando a categoria estabilidade financeira e as mulheres apontando a categoria não guardar mágoas como sendo importantes para o envelhecimento saudável. Tal resultado, pode ser explicado pela influência do contexto sócio-histórico da amostra estudada, no qual o homem tinha o papel de sustentação econômica da família, enquanto o aspecto afetivo e emocional eram mais compatíveis com o papel social desempenhado pela mulher que era a mãe e dona-decasa, demonstrando desta forma, a maior preocupação da mulher em estar melhor engajada, emocionalmente, no seu contexto de vida.

$\mathrm{Na}$ análise entre idade e a categoria saúde emocional: os grupos idoso-jovem e idoso-médio foram os que mais a indicaram, onde infere-se que para o idoso-idoso, há uma melhor elaboração cognitiva dessa fase da vida e mesmo uma não preocupação com alguns aspectos de sua vida, como por exemplo, ter reconhecimento de pessoas para sua satisfação pessoal, visto que as preocupações com os padrões e regras, instituídos pela cultura tendem a diminuir com o passar dos anos (Levenson, Aldwin \& Cupertino, 2001).

Outro ponto a ser considerado é a relação significativa entre o grupo idoso-jovem e a categoria alimentação $e$ exercícios físicos, o argumento relaciona-se a coorte histórica deste grupo, visto que somente nos últimos anos vê-se o movimento da mídia e sociedade em campanhas contra comportamentos de risco e incentivo a uma vida mais saudável (por meio, dentre outros aspectos, de atividades físicas e boa alimentação). A categoria estabilidade financeira também é uma preocupação maior por parte do idoso-jovem visto que, talvez, estes ainda estejam mais engajados profissionalmente do que os demais idosos da amostra.

É importante enfatizar que foram poucos que não conseguiram definir envelhecimento saudável bem como a forma de alcançá-lo, ou seja, nota-se que os idosos são capazes de visualizar o que seria o envelhecimento saudável, destacando-se apenas o grupo idoso-idoso que obtiveram dificuldades na exposição da definição. 
Tabela 3

Relação das Categorias Estratificada por Gênero e Idade

\begin{tabular}{|c|c|c|c|c|c|c|c|}
\hline \multirow[t]{2}{*}{ Categorias } & \multicolumn{3}{|c|}{ \% Gênero } & \multicolumn{4}{|c|}{$\%$ Idade } \\
\hline & Homem & Mulher & $x^{2}$ & $\begin{array}{l}\text { idoso- } \\
\text { jovem }\end{array}$ & $\begin{array}{l}\text { idoso- } \\
\text { médio }\end{array}$ & $\begin{array}{l}\text { idoso- } \\
\text { idoso }\end{array}$ & $x^{2}$ \\
\hline \multicolumn{8}{|l|}{ saúde social } \\
\hline $\operatorname{sim}$ & 12 & 34 & \multirow{2}{*}{$2.5 \mathrm{O}^{+}$} & 19 & 18 & 8 & \multirow{2}{*}{1.11} \\
\hline não & 17 & 37 & & 20 & 23 & 12 & \\
\hline \multicolumn{8}{|c|}{ saúde emocional } \\
\hline $\operatorname{sim}$ & 9 & 28 & \multirow{2}{*}{$2.74^{+}$} & 16 & 16 & 5 & \multirow{2}{*}{$7.72^{*}$} \\
\hline não & 20 & 43 & & 22 & 26 & 15 & \\
\hline \multicolumn{8}{|c|}{ alimentação e exercícios } \\
\hline $\operatorname{sim}$ & 11 & 25 & \multirow{2}{*}{0.06} & 16 & 13 & 6 & \multirow{2}{*}{$6.26^{*}$} \\
\hline não & 18 & 46 & & 22 & 29 & 14 & \\
\hline \multicolumn{8}{|c|}{ atividades gerais } \\
\hline $\operatorname{sim}$ & 4 & 14 & \multirow{2}{*}{1.08} & 8 & 8 & 2 & \multirow{2}{*}{$4.11^{+}$} \\
\hline não & 25 & 57 & & 31 & 33 & 18 & \\
\hline \multicolumn{8}{|c|}{ ter fé, espiritualidade } \\
\hline $\operatorname{sim}$ & 3 & 12 & \multirow{2}{*}{$3.43^{+}$} & 4 & 7 & 4 & \multirow{2}{*}{3.52} \\
\hline não & 26 & 59 & & 34 & 35 & 16 & \\
\hline \multicolumn{8}{|c|}{ atividade operacional } \\
\hline $\operatorname{sim}$ & 5 & 8 & \multirow{2}{*}{$3.31^{+}$} & 5 & 6 & 2 & \multirow{2}{*}{2.92} \\
\hline não & 24 & 63 & & 33 & 35 & 18 & \\
\hline \multicolumn{8}{|c|}{ estabilidade financeira } \\
\hline $\operatorname{sim}$ & 5 & 4 & \multirow{2}{*}{$21.8^{* * * *}$} & 5 & 3 & 1 & \multirow{2}{*}{$8.03 *$} \\
\hline não & 24 & 67 & & 33 & 38 & 19 & \\
\hline \multicolumn{8}{|c|}{ ter espírito jovem } \\
\hline $\operatorname{sim}$ & 1 & 4 & \multirow{2}{*}{$3.31^{+}$} & 1.6 & 3 & 0.4 & \multirow{2}{*}{3.60} \\
\hline não & 28 & 67 & & 37 & 39 & 19 & \\
\hline \multicolumn{8}{|c|}{ não guardar mágoas } \\
\hline $\operatorname{sim}$ & $\mathrm{O}$ & 4 & 6o $2 * *$ & 1 & 2 & 1 & 100 \\
\hline não & 29 & 67 & & 37 & 39 & 19 & 1.20 \\
\hline integridade & & & & & & & \\
\hline $\operatorname{sim}$ & $\mathrm{O}$ & 3 & o $5 \mathrm{O}^{+}$ & 2 & 1 & $\mathrm{O}$ & $550^{+}$ \\
\hline não & 29 & 68 & 2.02 & 36 & 41 & 20 & 5.50 \\
\hline não sabe de & & & & & & & \\
\hline $\operatorname{sim}$ & $\mathrm{O}$ & 1 & $900^{+}$ & $\mathrm{O}$ & 0.5 & 1 & $765 *$ \\
\hline não & 29 & 70 & 2.50 & 38.5 & 41 & 19 & 1.05 \\
\hline
\end{tabular}

Notas. ${ }^{*} * * p<0.001 ; * * * 0.01 ; * p<0.05 ;^{+} p<0.1$

Cabe ressaltar ainda a tendência de que as mulheres apontam em maior proporção as categorias saúde emocional e ter fé/espiritualidade do que os homens, donde infere-se que a mulher tende a uma maior preocupação com o bem-estar emocional do que os homens. Talvez elas visualizem o equilíbrio desses aspectos como essenciais para um bem-estar pessoal, enquanto os homens vislumbrem o bem-estar por meio de outros fatores, como uma adequada estabilidade financeira visto que estes exerciam o papel de provedor e mantenedor do lar como já justificado anteriormente. A categoria ter espírito jovem também é mais enfatizada pelas mulheres, indicando deste modo que as mulheres possuem maior dificuldade de aceitação do envelhecimento físico exterior do que os homens.

Desta forma, o envelhecimento saudável e suas multidimensões durante o curso da vida são temas amplos e pouco estudados, visto que muito investiga-se sobre o envelhecimento patológico e suas repercussões em diversos campos da vida do sujeito. A partir da coleta de dados sobre as perspectivas de envelhecimento saudável propostas pela literatura e, em especial pelo público-alvo, é possível haver uma maior integração e discussão sobre as possibilidades e características do que se considera como envelhecimento saudável. 


\section{Conclusão}

Este estudo confirmou os pressupostos de heterogeneidade e multidimensionalidade defendidos pela abordagem do curso da vida, pois observou-se variabilidade nas definições dos idosos, sendo várias as dimensões enfatizadas para a obtenção de um processo saudável de envelhecimento, tais como as dimensões física; social; emocional; econômica; cognitiva dentre outras.

Sugere-se que futuros estudos busquem desenvolver e validar escalas psicométricas que possibilitem a avaliação da opinião do idoso sobre o seu próprio envelhecimento e sobre as várias dimensões relacionadas ao processo de envelhecimento de forma a ampliar o conhecimento teórico das variáveis determinantes de um processo saudável de envelhecimento e contribuindo para melhorar a qualidade e eficácia do atendimento às necessidades da população idosa.

Conclui-se que o presente trabalho contribui para a compreensão de como a população idosa define o envelhecimento. Entretanto, futuros trabalhos de coorte seqüencial são necessários em prol da avaliação sistemática se a relação entre as categorias na definição de envelhecimento saudável, gênero e idade podem ser realmente explicadas isoladamente ou seriam melhores justificadas pelo contex to sócio-histórico da amostra.

\section{Referências}

Aldwin, C. M. (1994). Stress, coping and development: An interative. New York: Guilford.

Baltes, P. B., \& Baltes, M. M. (1990). Psychological perspectives on sucessful aging: The model of selective optimization with compensation. In P. B. Baltes \& M. M. Baltes (Eds.), Sucessful aging: Perspectives from the Behavioral Sciences (pp. 1-34). New York: Cambridge University Press.
Baltes, P. B., \& Smith, J. (1995). Psicologia da sabedoria: Origem e desenvolvimento (A. L. Neri \& L. Goldstein, Trad.). In A. L. Neri (Ed.), Psicologia do envelhecimento (pp. 41-72). Campinas, SP: Papirus

Bardin, L. (1979). Análise de conteúdo (L. A. Reto \& A. Pinheiro, Trad.). São Paulo, SP: Edições 70/Livraria Martins Fontes. (Original publicado em 1977)

Levenson, M., Aldwin, M., \& Cupertino, A. P. (2001) Transcendendo o ego: Um modelo liberador. In A. L. Neri. Maturidade e velhice (pp. 99-115) Campinas, SP: Papirus.

Mauad, L. C. (1999). Sabedoria e revisão de vida: O desempenho de um grupo de mulheres de diferentes idades. Londrina, PR: Editora da Universidade Estadual de Londrina.

Neri, A. L. (1997). A pesquisa em Gerontologia no Brasil. Análise de conteúdos de amostra de pesquisa em psicologia no período de 1975-1976. Texto Contexto Enfermagem, Florianópolis, v. 6, n. $2,69-105$.

Papalia, D. E., \& Olds, S. W. (2000). Desenvolvimento humano. Porto Alegre, RS: Artmed.

Paschoal, S. M. P. (2002) Qualidade de vida na velhice. In E. V. Freitas, L. Py, A. L. Neri, F. A. X. Cançado, M. L. Gorzoni, S. M. Rocha (Eds.), Tratado de Geriatria e Gerontologia (pp. 7984). Rio de Janeiro, RJ: Guanabara Koogan.

Ramos, L. R. (2002) Epidemiologia do envelhecimento. In E. V. Freitas, L. Py, A. L. Neri, F. A. X. Cançado, M. L. Gorzoni, S. M. Rocha (Eds.), Tratado de Geriatria e Gerontologia (pp. 7278). Rio de Janeiro, RJ: Guanabara Koogan.

Rowe, J. W., \& Kahn, R. L (1998). Successful aging. New York: Pantenon Books.

Staudinger, U. M, Marsisker, M., \& Baltes, P. B. (1995) Resiliência e níveis de capacidade de reserva na velhice: Perspectivas da teoria de curso da vida. In A. L. Neri (Ed.), Psicologia do envelhecimento (pp. 195-228). Campinas, SP: Papirus.

Recebido: 26/05/2005

$1^{a}$ revisão: 06/12/2005

Aceite final. 10/02/2006 